\title{
Histopathological and Genetic Features of Patients with Limb Girdle Muscular Dystrophy Type 2C
}

\author{
Kavşak Tipi Müsküler Distrofi Tip 2C Hastalarının Histopatolojik ve \\ Genetik Özellikleri
}

\section{Gülden DiNiZ1', Filiz HAZAN², Hülya TOSUN YILDIRIM³, Aycan ÜNALP', Muzaffer POLAT5,

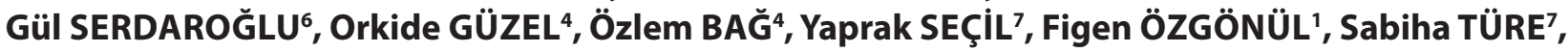 Galip AKHAN ${ }^{7}$, Ajlan TÜKÜN8}

\author{
${ }^{1}$ Tepecik Education and Research Hospital, Neuromuscular Disease Center, IZMIR, TURKEY \\ Department of ${ }^{2}$ Medical Genetics, ${ }^{3}$ Pathology and ${ }^{4}$ Pediatric Neurology, Dr. Behçet Uz Children's Hospital, iZMiR, TURKEY \\ Department of Pediatric Neurology ${ }^{5}$ Celal Bayar University, Faculty of Medicine, MANISA, TURKEY and ${ }^{6}$ Ege University, Faculty of Medicine, iZMiR, TURKEY \\ ${ }^{7}$ Department of Neurology, Katip Çelebi University, Faculty of Medicine, IZMIR, TURKEY \\ ${ }^{8}$ Department of Medical Genetics, Düzen Laboratory, ANKARA, TURKEY
}

\begin{abstract}
Objective: In this study, it was aimed to describe the clinical, histopathological and genetic features of 20 patients with gamma sarcoglycanopathy confirmed by muscle biopsies and genetic analysis.

Material and Method: We retrospectively reviewed 20 patients from whom muscle biopsy specimens were obtained between 2007 and 2012. All patients were clinically diagnosed as muscular dystrophy and biopsy materials were collected from five different centers of neurological disorders. All DNAs were extracted from muscle tissues or blood samples of patients and genetic tests (mutation analyses for gamma sarcoglycan gene and deletion-duplication analyses for all 4 sarcoglycan genes) were performed.
\end{abstract}

Results: The mean age of the patients was 7.6 years (2 -21 years). Only one case (5\%) was older than 14 years. The mean CPK level was $10311 \mathrm{U} / \mathrm{L}$ (1311 - $35000 \mathrm{U} / \mathrm{L})$. There were 4 siblings in these series. Expression defects of gamma sarcoglycan staining were determined in (15 males, and 5 females) all patients with muscle biopsy specimens. But only in 9 of them, disease-causing defects could be determined with genetic analyses.

Conclusion: The present study has demonstrated that both examination of muscle biopsy specimens and DNA analysis remain important methods in the differential diagnosis of muscular dystrophies. Because dystrophinopathies and sarcoglycanopathies have similar clinical manifestation.

Key Words: Dystrophin, Gamma sarcoglycan, Genetic testing, Muscular dystrophy, Limb-Girdle

\section{ÖZ}

Amaç: Bu çalışmada, kas biyopsi incelemesi ve genetik analizle tanısı doğrulanan gama sarkoglikanopatili 20 olgunun klinik, histopatolojik ve genetik özelliklerinin sunulması amaçlandı.

Gereç ve Yöntem: Patoloji laboratuvarımızda, 2007 ile 2012 yılları arasında kas biyopsisi incelenen 20 hasta retrospektif olarak değerlendirildi. Tüm hastalar klinik olarak müsküler distrofi tanısı almış olup, biyopsiler 5 farklı nöroloji kliniğinde alındı. Hasta DNA'ları kas dokusu veya periferik kandan çıkartıldı ve genetik testler (gama sarkoglikan geni için mutasyon analizleri ve tüm 4 sarkoglikan için delesyon duplikasyon testleri) yapıldı.

Bulgular: Hastaların ortalama yaşı 7,6 yıl (2- 21 yıl) idi. Yalnızca tek olgu (\%5) 14 yaşın üzerindeydi. Ortalama CPK düzeyleri 10311 U/L (1311- 35000 U/L) bulundu. Grupta 4 kardeş hasta $(n=8)$ vardı. Kas biyopsisinde tüm hastalarda (15 erkek ve 5 kadın) gama sarkoglikan boyanma defekti saptandi. Fakat genetik incelemede sadece 9 hastada gama sarkoglikan geninde hastalığa yol açacak defekt vardı.

Sonuç: Çalışmamız, hem kas biyopsi incelemesi, hem de genetik analizin müsküler distrofilerin ayırıcı tanısında çok önemli olduğunu göstermiştir. Çünkü distrofinopati ve sarkoglikanopatiler benzer klinik bulgulara sahiptirler.

Anahtar Sözcükler: Distrofin, Gama sarkoglikan, Genetik analiz, Müsküler distrofi, Uzuv-Kemer

Correspondence: Gülden DİNiZ

Tepecik Eğitim ve Araştırma Hastanesi, Nöromuskuler Hastalıklar Merkezi, IZMİR, TURKEY

E-mail: agdiniz@gmail.com Phone: +90 2324696969 


\section{INTRODUCTION}

Gamma sarcoglycan ( $\gamma$-SGC) is one of the four sarcoglycans (SGCs) found at the cell membrane of skeletal muscle. The SGCs form a subcomplex closely linked to the dystrophinassociated glycoprotein complex (DAG). Proper presence of SGCs is essential for membrane integrity during muscle contraction. Limb girdle muscular dystrophy type 2C (LGMD-2C) is an autosomal recessive muscle-wasting disorder caused by genetic defects in the sarcoglycan gamma (SGCG) gene. It is also known as the childhood severe muscular dystrophy and clinically resembles the dystrophinopathies which are the most common muscular dystrophies (1-4).

No definitive treatments for the LGMD-2C and the other muscular dystrophies exist. Management to prolong survival and improve quality of life includes physical therapy, and stretching exercises to promote mobility and prevent contractures, weight control to avoid obesity, surgery for orthopedic complications, use of mechanical and respiratory aids to help ambulation, mobility and respiration. Monitoring cardiomyopathy for cardiac involvement and emotional support are also required (5-9).

Differential diagnosis of LGMD-2C is made in consideration of Duchenne and Becker muscular dystrophies $(\mathrm{DMD} / \mathrm{BMD})$ and it is impossible to differentiate between these conditions solely on clinical grounds. Therefore immunohistochemical staining of muscle biopsy specimens and molecular genetic analysis are mandatory for correct diagnosis (10-12). In this study, we aimed to determine the spectrum of genetic defects in immunohistochemically proven cases of LGMD-2C, to correlate the findings with clinical phenotypes and to display the regional differences as for the clinical, histopathological, and genetic characteristics of gamma sarcoglycanopathies.

\section{MATERIAL and METHODS}

Histopatological examinations of muscle biopsies were performed at Pathology Laboratory of Izmir Dr. Behçet Uz Children's Hospital. Genetic analyses were performed at Ankara Düzen Laboratory from January 2007 through December 2012. Twenty patients with defective gamma sarcoglycan expressions found on the muscle biopsy specimens, and clinically diagnosed as muscular dystrophy were included in this study. Immunohistochemical analysis (IHC) was repeated to confirm the diagnosis. Individual patient database was reviewed in all cases, and clinical information of patients was recorded including age, gender, detailed family history and consanguinity. Neurological examination and laboratory findings were also evaluated.
Laboratory evaluation included serum creatine kinase (CK), serum aspartate aminotransferase (AST) analyses, and nerve conduction and electromyographic (EMG) studies. All muscle biopsies were obtained from the gastrocnemius muscle.

Samples were frozen in isopentane cooled in liquid nitrogen and 8- to 12- micron sections were cut using the cryostat. Slides were stained with hematoxylin-eosin (H\&E), as well as with several histochemical and enzymatic stains. Cryosections were immunostained for dystrophin using a polyclonal antibody (Neomarkers) with a monoclonal spectrin antibody (Novocastra) as a control. SGCs were detected with anti alpha $(\alpha-)$, beta $(\beta-)$, delta $(\delta-)$ and $\gamma-$ SGC antibodies (Novocastra).

Genomic DNAs were extracted from the remnant muscle tissues or blood samples using available DNA extraction kits (QiaGen, US) following the manufacturer's standard protocol. The exon regions and flanking short intronic sequences of the SGCG gene were amplified using the polymerase chain reaction (PCR), followed by direct sequencing of the $\mathrm{PCR}$ products (ABI, US). In addition, the multiplex ligation-dependent probe amplification (MLPA) technique was used for deletion and duplication analysis for all 4 SGCs.

Frequencies and descriptive analyses were performed using the statistical software SPSS 9.05 for Windows.

\section{RESULTS}

Twenty patients with severe muscle disease were evaluated respectively. All of them had been diagnosed as muscular dystrophy on the basis of muscle biopsy findings. Severe alterations of myofiber size and shape, splitting, increase in the number of internal nuclei, fiber type disproportions; necrosis, myophagocytosis, regeneration and fibrosis were simply classified as muscular dystrophy (Figure 1).

The mean age of the patients was 7.6 years (2 to 21 years). There were 4 siblings $(n=8)$ in these series. Expression defects of gamma sarcoglycan staining were determined in (15 male and 5 female) all patients with available muscle biopsy specimens. However, disease-causing defects could be determined with genetic analyses in only 9 of them. The mean age of the patients was 7.6 years $( \pm 4.11)$, ranging from 2 to 21 years. Only one case (5\%) was older than 14 years. The detailed clinical characteristics of the patients were presented in Table I. All patients presented some degree of muscle weakness. All of them had high creatine kinase (CPK) levels. The mean CPK level was 10311 U/L (1311 $35000 \mathrm{U} / \mathrm{L}$ ). Ten patients (50\%) had similarly affected family 


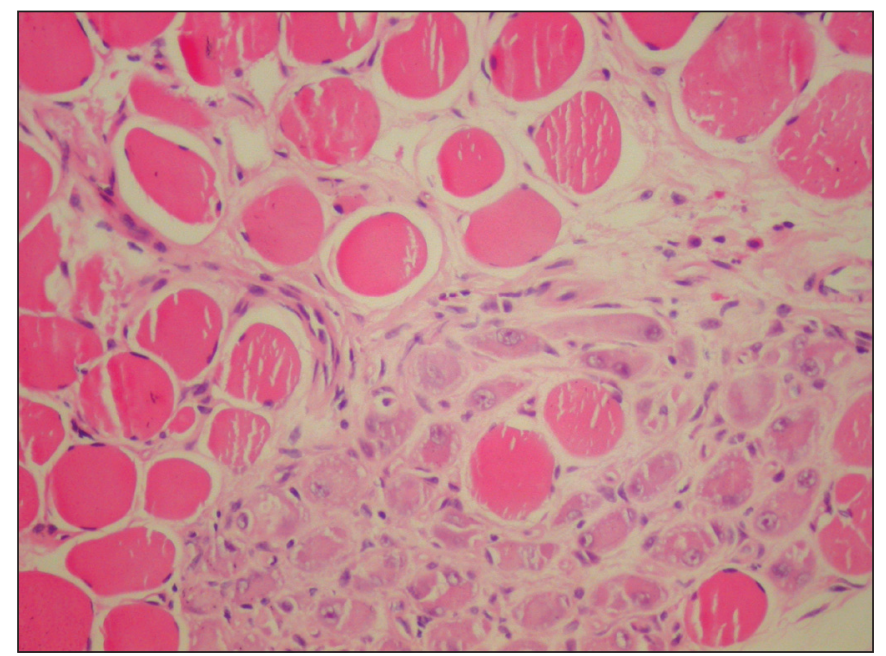

Figure 1: Note the regenerated muscle fibers which are specific for muscular dystrophies (H\&E, x100).
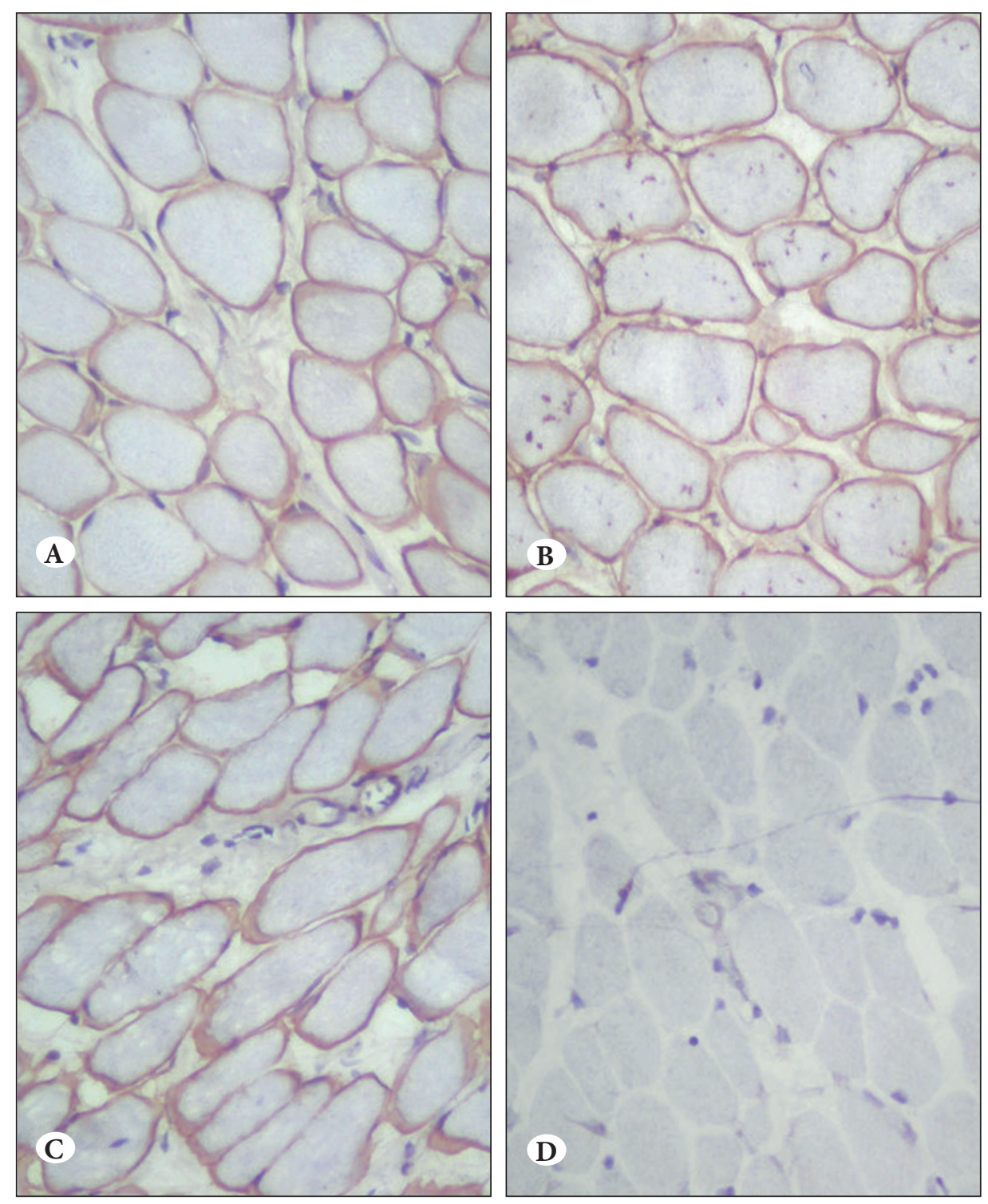

Figure 2: A) Nearly normal expressions of $\alpha$-SGC, B) $\beta$-SGC, C) $\delta$-SGC and D) Defective expression of sarcolemmal $\gamma$-SGC (DAB, x100). 
Table I: Clinical and genetic features of patients

\begin{tabular}{|c|c|c|c|c|c|c|c|c|c|c|}
\hline נֶ & $\stackrel{\varpi}{\infty}$ & $\stackrel{\square}{<}$ & 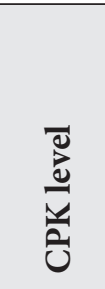 & 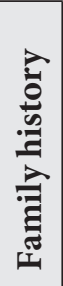 & 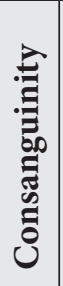 & 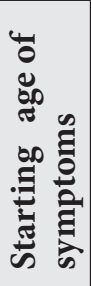 & 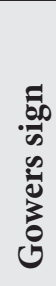 & 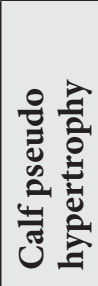 & 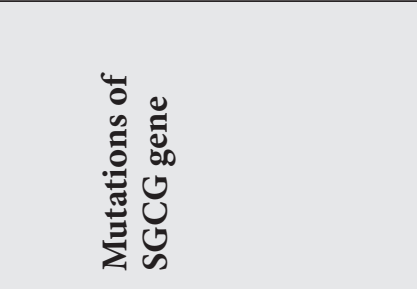 & 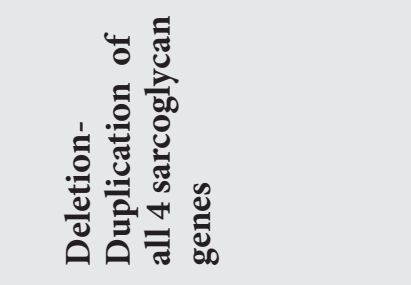 \\
\hline 1 & $\mathrm{~F}$ & 21 & 5300 & - & - & 8 & + & - & $\begin{array}{l}\text { R116H(G347A), } \\
\text { S287N(G860A), } \\
\text { I218T(T654C) Compound } \\
\text { heterozygosity }\end{array}$ & - \\
\hline 2 & $\mathrm{M}$ & 6 & 4500 & - & - & 2.5 & + & + & $\begin{array}{l}\text { R116H(G347A), } \\
\text { S287N(G860A), } \\
\text { Compound heterozygosity }\end{array}$ & - \\
\hline 3 & $\mathrm{M}$ & 9 & 15000 & + & + & 7 & - & - & - & - \\
\hline 4 & $\mathrm{M}$ & 11 & 17500 & + & - & 6 & + & + & S287N(G860A) & - \\
\hline 5 & $\mathrm{M}$ & 11 & 5000 & - & - & 8 & + & + & R116H(G347A) & $\begin{array}{l}\text { Duplication of exon } 10 \text { in } \\
\text { SGCA gene }\end{array}$ \\
\hline 6 sibling1 & $\mathrm{M}$ & 8 & 2200 & + & - & 5 & - & + & R116H(G347A) & - \\
\hline 7 sibling 1 & $\mathrm{M}$ & 5 & 6100 & + & - & 4 & + & + & $\mathrm{R} 116 \mathrm{H}(\mathrm{G} 347 \mathrm{~A})$ & - \\
\hline 8 sibling 2 & $\mathrm{M}$ & 6 & 5000 & + & - & 5 & + & + & 808-801DELGT & SGCG.Exon8 LOH \\
\hline 9 sibling 2 & $\mathrm{M}$ & 8 & 13000 & + & - & 4 & + & + & 808-801DELGT & SGCG.Exon8 LOH \\
\hline 10 & $\mathrm{~F}$ & 7 & 10000 & - & + & 5 & - & - & $\begin{array}{l}\text { R116H(G347A), } \\
\text { S287N(G860A), }\end{array}$ & $\begin{array}{l}\text { Duplication of exons 1-10 } \\
\text { in SGCA gene }\end{array}$ \\
\hline 11 & $\mathrm{M}$ & 10 & 7500 & - & + & 7 & + & + & - & $\begin{array}{l}\text { İntragenic amplification in } \\
\text { SGCA gene }\end{array}$ \\
\hline 12 & $\mathrm{M}$ & 6 & 11000 & - & + & 9 & + & + & - & - \\
\hline 13 & $\mathrm{M}$ & 10 & 7500 & - & - & 9 & - & - & T312G, T705C & - \\
\hline 14 & $\mathrm{M}$ & 2 & 1312 & - & - & 2 & - & - & $\mathrm{R} 116 \mathrm{H}(\mathrm{G} 347 \mathrm{~A})$ & - \\
\hline 15 & $\mathrm{M}$ & 7 & 11000 & - & + & 6 & + & + & T312G, T705C & - \\
\hline 16 sibling 3 & $\mathrm{~F}$ & 2 & 7541 & + & + & 2 & - & - & 525delT homozygous & - \\
\hline 17 sibling 3 & $\mathrm{M}$ & 6 & 15784 & + & + & 5 & + & - & 525delT homozygous & - \\
\hline 18 & $\mathrm{~F}$ & 3 & 13000 & - & - & 2 & - & - & T312G, T705C & - \\
\hline 19 sibling4 & $\mathrm{F}$ & 6 & 35000 & + & + & 4 & + & + & T228C, T312G, T705C & - \\
\hline 20 sibling 4 & $\mathrm{M}$ & 8 & 13000 & + & + & 4 & + & + & T228C, T312G, T705C & - \\
\hline
\end{tabular}

\section{DISCUSSION}

The human SGCG gene is located on chromosome 13q12. It consists of 8 exons. The sequence of SGCG is composed of 291 amino acids. Three portions of the gamma sarcoglycan extracellular domain display possible critical function, two for the assembly with either beta or alpha sarcoglycan, and the putative EGF-like domain. Hitherto forty mutations have been described in the gamma sarcoglycan gene (2). The homozygous del525T mutation generates a truncated gamma sarcoglycan protein without EGF-like domain, which is able to assemble with the other sarcoglycans $(1,2,13-15)$. This mutation is commonly found in North Africa. The C283Y mutation in the cystein-rich domain could be functionally relevant, because this cysteine is crucial for the EGF-like domain. The C283Y mutation can cause severe LGMD and it is the most common mutation in the Gypsy ethnic groups of the Europe $(1,2,15-17)$. In the present study, we have determined homozygous del525T mutation in a sibling, but we could not find a C283Y mutation. 
Table II: Nucleotide and amino acid sequences of SGCG gene

\begin{tabular}{|c|c|c|c|c|c|c|c|c|c|c|c|c|c|c|c|c|}
\hline $\begin{array}{l}1 \\
1\end{array}$ & $\begin{array}{l}\text { G } \\
\text { et }\end{array}$ & $\begin{array}{l}\text { GTG } \\
\text { Val }\end{array}$ & $\begin{array}{c}\text { CGT } \\
\text { Arg }\end{array}$ & $\begin{array}{c}\text { GAG } \\
\text { Glu }\end{array}$ & $\begin{array}{l}\text { CAG } \\
\text { Gln }\end{array}$ & $\begin{array}{l}\text { TAC } \\
\text { Tyr }\end{array}$ & $\begin{array}{c}\text { ACT } \\
\text { Thr }\end{array}$ & $\begin{array}{l}\text { ACA } \\
\text { Thr }\end{array}$ & $\begin{array}{l}\text { CC } \\
\text { lla }\end{array}$ & $\begin{array}{c}\text { ACA } \\
\text { Thr }\end{array}$ & $\begin{array}{c}\text { GAA } \\
\text { Glu }\end{array}$ & $\begin{array}{l}\text { GGC } \\
\text { Gly }\end{array}$ & $\begin{array}{l}\text { ATC } \\
\text { Ile }\end{array}$ & $\begin{array}{l}\text { TGC } \\
\text { Cys }\end{array}$ & $\begin{array}{l}\text { ATA } \\
\text { Ile }\end{array}$ & $\begin{array}{l}45 \\
15\end{array}$ \\
\hline $\begin{array}{l}46 \\
16\end{array}$ & $\begin{array}{l}\text { GAG } \\
\text { Glu }\end{array}$ & $\begin{array}{c}\text { AGG } \\
\text { Arg }\end{array}$ & $\begin{array}{c}\text { CCA } \\
\text { Pro }\end{array}$ & $\begin{array}{c}\text { GAG } \\
\text { Glu }\end{array}$ & $\begin{array}{l}\text { AAT } \\
\text { Asn }\end{array}$ & $\begin{array}{c}\text { CAG } \\
\text { Gln }\end{array}$ & Tyr & $\begin{array}{c}\text { GTC } \\
\text { Val }\end{array}$ & Tyr & Lys & $\begin{array}{l}\text { ATT } \\
\text { Ile }\end{array}$ & $\begin{array}{l}\text { GGC } \\
\text { Gly }\end{array}$ & $\begin{array}{l}\text { ATT } \\
\text { Ile }\end{array}$ & $\begin{array}{l}\text { TAT } \\
\text { Tyr }\end{array}$ & $\begin{array}{l}\text { GGC } \\
\text { Gly }\end{array}$ & $\begin{array}{l}90 \\
30\end{array}$ \\
\hline $\begin{array}{l}91 \\
31 \\
\end{array}$ & $\begin{array}{l}\mathrm{G} \\
\mathrm{p}\end{array}$ & $\begin{array}{c}\text { AGA } \\
\text { Arg }\end{array}$ & $\begin{array}{c}\text { AAG } \\
\text { Lys }\end{array}$ & $\begin{array}{c}\text { CGC } \\
\text { Arg }\end{array}$ & 0 & $\begin{array}{l}\text { CTC } \\
\text { Leu }\end{array}$ & Tyr & $\begin{array}{l}\text { TTG } \\
\text { Leu }\end{array}$ & e & Val & $\begin{array}{l}\text { ГT } \\
\mathrm{eu}\end{array}$ & $\mathrm{T}$ & $\begin{array}{l}\text { TA } \\
\text { eu }\end{array}$ & $\begin{array}{c}\text { CTC } \\
\text { Leu }\end{array}$ & $\begin{array}{l}\text { ATC } \\
\text { Ile }\end{array}$ & $\begin{array}{c}135 \\
45 \\
\end{array}$ \\
\hline $\begin{array}{c}136 \\
46 \\
\end{array}$ & $\begin{array}{c}\text { ATC } \\
\text { Ile }\end{array}$ & $\begin{array}{c}\text { CTC } \\
\text { Leu }\end{array}$ & $\begin{array}{c}\text { GTT } \\
\text { Val }\end{array}$ & $\begin{array}{c}\text { GTG } \\
\text { Val }\end{array}$ & $\begin{array}{l}\text { AAT } \\
\text { Asn }\end{array}$ & $\begin{array}{l}\text { TTA } \\
\text { Leu }\end{array}$ & $\begin{array}{c}\text { GCT } \\
\text { Ala }\end{array}$ & $\begin{array}{c}\text { CTT } \\
\text { Leu }\end{array}$ & $\begin{array}{l}\text { ACA } \\
\text { Thr }\end{array}$ & $\begin{array}{c}\text { ATT } \\
\text { Ile }\end{array}$ & $\begin{array}{l}\text { TGG } \\
\text { Trp }\end{array}$ & $\begin{array}{c}\text { ATT } \\
\text { Ile }\end{array}$ & $\begin{array}{l}\text { CTT } \\
\text { Leu } \\
\end{array}$ & $\begin{array}{c}\text { AAA } \\
\text { Lys }\end{array}$ & $\begin{array}{c}\text { GTG } \\
\text { Val }\end{array}$ & $\begin{array}{c}180 \\
60 \\
\end{array}$ \\
\hline $\begin{array}{c}181 \\
61\end{array}$ & $\begin{array}{c}\text { ATG } \\
\text { Met }\end{array}$ & $\begin{array}{l}\text { TGG } \\
\text { Trp }\end{array}$ & $\begin{array}{l}\text { TTT } \\
\text { Phe }\end{array}$ & $\begin{array}{l}\text { TCT } \\
\text { Ser }\end{array}$ & $\begin{array}{c}\text { CCA } \\
\text { Pro }\end{array}$ & $\begin{array}{c}\text { GCA } \\
\text { Ala }\end{array}$ & $\begin{array}{c}\text { GGA } \\
\text { Gly }\end{array}$ & $\begin{array}{c}\text { ATG } \\
\text { Met }\end{array}$ & $\begin{array}{c}\text { GGC } \\
\text { Gly }\end{array}$ & $\begin{array}{c}\text { CAC } \\
\text { His }\end{array}$ & $\begin{array}{l}\text { TTG } \\
\text { Leu }\end{array}$ & $\begin{array}{l}\text { TGT } \\
\text { Cys }\end{array}$ & $\begin{array}{c}\text { GTA } \\
\text { Val }\end{array}$ & $\begin{array}{c}\text { ACA } \\
\text { Thr }\end{array}$ & $\begin{array}{c}\text { AAA } \\
\text { Lys }\end{array}$ & $\begin{array}{c}225 \\
75 \\
\end{array}$ \\
\hline $\begin{array}{c}226 \\
76\end{array}$ & $\begin{array}{l}\text { SAT } \\
\text { Asp }\end{array}$ & $\begin{array}{c}\text { GGA } \\
\text { Gly }\end{array}$ & $\begin{array}{l}\text { CTG } \\
\text { Leu }\end{array}$ & $\begin{array}{c}\text { CGC } \\
\text { Arg }\end{array}$ & $\begin{array}{l}\text { TTG } \\
\text { Leu }\end{array}$ & $\begin{array}{c}\text { GAA } \\
\text { Glu }\end{array}$ & $\begin{array}{c}\text { GGG } \\
\text { Gly }\end{array}$ & $\begin{array}{c}\text { GAA } \\
\text { Glu }\end{array}$ & $\begin{array}{l}\text { TCA } \\
\text { Ser }\end{array}$ & $\begin{array}{c}\text { GAA } \\
\text { Glu }\end{array}$ & $\begin{array}{l}\text { TTT } \\
\text { Phe }\end{array}$ & $\begin{array}{l}\text { TTA } \\
\text { Leu }\end{array}$ & $\begin{array}{l}\text { TTC } \\
\text { Phe }\end{array}$ & $\begin{array}{c}\text { CCA } \\
\text { Pro }\end{array}$ & $\begin{array}{l}\text { TTG } \\
\text { Leu }\end{array}$ & $\begin{array}{c}270 \\
90\end{array}$ \\
\hline $\begin{array}{c}271 \\
91\end{array}$ & $\begin{array}{l}\text { TAT } \\
\text { Tyr }\end{array}$ & $\begin{array}{c}\text { GCC } \\
\text { Ala }\end{array}$ & $\begin{array}{c}\text { AAA } \\
\text { Lys }\end{array}$ & & $\begin{array}{l}\text { ATA } \\
\text { Ile }\end{array}$ & $\begin{array}{c}\text { CAC } \\
\text { His }\end{array}$ & $\begin{array}{l}\text { TCC } \\
\text { Ser }\end{array}$ & $\begin{array}{c}\text { AGA } \\
\text { Arg }\end{array}$ & $\begin{array}{c}\text { GTG } \\
\text { Val }\end{array}$ & $\begin{array}{c}\text { GAC } \\
\text { Asp }\end{array}$ & $\begin{array}{l}\text { TCA } \\
\text { Ser }\end{array}$ & $\begin{array}{l}\text { TCT } \\
\text { Ser }\end{array}$ & $\begin{array}{l}\text { CTG } \\
\text { Leu }\end{array}$ & $\frac{\mathrm{CTT}}{\mathrm{Leu}}$ & $\begin{array}{l}\text { CTA } \\
\text { Leu }\end{array}$ & $\begin{array}{l}315 \\
105\end{array}$ \\
\hline $\begin{array}{l}316 \\
106\end{array}$ & & $\begin{array}{l}\text { TCA } \\
\text { Ser }\end{array}$ & $\begin{array}{l}\text { ACC } \\
\text { Thr }\end{array}$ & & & $\begin{array}{c}\text { GTG } \\
\text { Val }\end{array}$ & $\begin{array}{c}\text { ACT } \\
\text { Thr }\end{array}$ & $\begin{array}{c}\text { GTA } \\
\text { Val }\end{array}$ & & & $\begin{array}{l}\text { CGC } \\
\text { Arg }\end{array}$ & & $\begin{array}{l}\text { TCA } \\
\text { Ser }\end{array}$ & $\begin{array}{c}\text { GAA } \\
\text { Glu }\end{array}$ & $\begin{array}{c}\text { GGG } \\
\text { Gly }\end{array}$ & $\begin{array}{l}360 \\
120\end{array}$ \\
\hline $\begin{array}{l}361 \\
121 \\
\end{array}$ & & $\begin{array}{c}\text { GTC } \\
\text { Val }\end{array}$ & $\begin{array}{c}\text { ACA } \\
\text { Thr }\end{array}$ & $\begin{array}{c}\text { GGC } \\
\text { Gly }\end{array}$ & & $\begin{array}{l}\text { TTA } \\
\text { Leu }\end{array}$ & $\begin{array}{c}\text { AAA } \\
\text { Lys }\end{array}$ & $\begin{array}{c}\text { GTC } \\
\text { Val }\end{array}$ & & $\begin{array}{c}\text { CCC } \\
\text { Pro } \\
\end{array}$ & $\begin{array}{c}\text { AAA } \\
\text { Lys }\end{array}$ & & $\begin{array}{c}\text { GTA } \\
\text { Val }\end{array}$ & $\begin{array}{c}\text { GAA } \\
\text { Glu }\end{array}$ & $\begin{array}{c}\text { GTC } \\
\text { Val }\end{array}$ & $\begin{array}{l}405 \\
135 \\
\end{array}$ \\
\hline $\begin{array}{l}406 \\
136 \\
\end{array}$ & $\begin{array}{c}\text { CAG } \\
\text { Gln }\end{array}$ & $\begin{array}{c}\text { AAT } \\
\text { Asn }\end{array}$ & $\begin{array}{c}\text { CAA } \\
\text { Gln }\end{array}$ & $\begin{array}{c}\text { CAG } \\
\text { Gln }\end{array}$ & & $\begin{array}{c}\text { CAG } \\
\text { Gln }\end{array}$ & $\begin{array}{c}\text { ATC } \\
\text { Ile }\end{array}$ & $\begin{array}{c}\text { AAC } \\
\text { Asn }\end{array}$ & $\begin{array}{c}\text { TCC } \\
\text { Ser }\end{array}$ & $\begin{array}{c}\text { AAC } \\
\text { Asn }\end{array}$ & $\begin{array}{c}\text { GAC } \\
\text { Asp }\end{array}$ & $\begin{array}{c}\text { GGC } \\
\text { Gly }\end{array}$ & $\begin{array}{l}\text { AAG } \\
\text { Lys }\end{array}$ & $\begin{array}{c}\text { CCA } \\
\text { Pro }\end{array}$ & $\begin{array}{c}\text { CTA } \\
\text { Leu }\end{array}$ & $\begin{array}{l}450 \\
150 \\
\end{array}$ \\
\hline $\begin{array}{l}451 \\
151\end{array}$ & $\begin{array}{l}\text { TTT } \\
\text { Phe }\end{array}$ & $\begin{array}{c}\text { ACT } \\
\text { Thr }\end{array}$ & $\begin{array}{c}\text { GTA } \\
\text { Val }\end{array}$ & & Glu & $\begin{array}{c}\text { AAG } \\
\text { Lys }\end{array}$ & $\begin{array}{c}\text { GAA } \\
\text { Glu }\end{array}$ & $\begin{array}{c}\text { GTT } \\
\text { Val }\end{array}$ & $\begin{array}{c}\text { GTG } \\
\text { Val }\end{array}$ & $\begin{array}{c}\text { GTT } \\
\text { Val }\end{array}$ & $\begin{array}{l}\text { GGT } \\
\text { Gly }\end{array}$ & $\begin{array}{c}\text { ACA } \\
\text { Thr }\end{array}$ & $\begin{array}{l}\text { GAT } \\
\text { Asp }\end{array}$ & $\begin{array}{l}\text { AAA } \\
\text { Lys }\end{array}$ & $\begin{array}{l}\text { CTT } \\
\text { Leu }\end{array}$ & $\begin{array}{l}495 \\
165 \\
\end{array}$ \\
\hline $\begin{array}{l}496 \\
166 \\
\end{array}$ & Arg & $\begin{array}{c}\text { GTA } \\
\text { Val }\end{array}$ & $\begin{array}{c}\text { ACT } \\
\text { Thr }\end{array}$ & $\begin{array}{c}\text { GGG } \\
\text { Gly }\end{array}$ & $\operatorname{Pr}$ & $\begin{array}{c}\text { GAA } \\
\text { Glu }\end{array}$ & $\begin{array}{c}\text { GGG } \\
\text { Gly }\end{array}$ & $\begin{array}{c}\text { GCT } \\
\text { Ala }\end{array}$ & & Phe & $\begin{array}{c}\text { GAA } \\
\text { Glu }\end{array}$ & $\begin{array}{c}\text { CAT } \\
\text { His }\end{array}$ & $\begin{array}{l}\text { TCA } \\
\text { Ser }\end{array}$ & $\begin{array}{c}\text { GTG } \\
\text { Val }\end{array}$ & $\begin{array}{c}\text { GAG } \\
\text { Glu }\end{array}$ & $\begin{array}{l}540 \\
180 \\
\end{array}$ \\
\hline $\begin{array}{l}54 \\
18\end{array}$ & & Pro & $\begin{array}{l}\text { CTT } \\
\text { Leu }\end{array}$ & $\begin{array}{c}\text { GTC } \\
\text { Val }\end{array}$ & & $\begin{array}{c}\text { GCC } \\
\text { Ala }\end{array}$ & $\begin{array}{c}\text { GAC } \\
\text { Asp }\end{array}$ & Pro & & $\begin{array}{c}\text { CAA } \\
\text { Gln }\end{array}$ & $\begin{array}{c}\text { GAC } \\
\text { Asp }\end{array}$ & Leu & Arg & $\begin{array}{l}\text { TTA } \\
\text { Leu }\end{array}$ & $\begin{array}{c}\text { GAA } \\
\text { Glu }\end{array}$ & 195 \\
\hline $\begin{array}{l}586 \\
196 \\
\end{array}$ & $\begin{array}{c}\text { TCC } \\
\text { Ser }\end{array}$ & $\begin{array}{c}\text { CCC } \\
\text { Pro }\end{array}$ & $\begin{array}{c}\text { ACT } \\
\text { Thr }\end{array}$ & $\begin{array}{c}\text { CGG } \\
\text { Arg }\end{array}$ & Ser & $\begin{array}{c}\text { CTA } \\
\text { Leu }\end{array}$ & $\begin{array}{c}\text { AGC } \\
\text { Ser }\end{array}$ & Met & & $\begin{array}{c}\text { GCC } \\
\text { Ala } \\
\end{array}$ & $\begin{array}{c}\text { CCA } \\
\text { Pro }\end{array}$ & $\begin{array}{c}\text { AGG } \\
\text { Arg }\end{array}$ & $\begin{array}{c}\text { GGT } \\
\text { Gly }\end{array}$ & $\begin{array}{c}\text { GTG } \\
\text { Val }\end{array}$ & $\begin{array}{c}\text { CAT } \\
\text { His }\end{array}$ & $\begin{array}{l}630 \\
210 \\
\end{array}$ \\
\hline $\begin{array}{l}631 \\
211\end{array}$ & $\begin{array}{l}\text { ATT } \\
\text { Ile }\end{array}$ & $\begin{array}{c}\text { CAA } \\
\text { Gln }\end{array}$ & $\begin{array}{c}\text { GCT } \\
\mathrm{Ala}\end{array}$ & $\begin{array}{c}\text { CAC } \\
\text { His }\end{array}$ & $\begin{array}{c}\text { GCT } \\
\mathrm{Ala}\end{array}$ & $\begin{array}{c}\text { GGG } \\
\text { Gly }\end{array}$ & $\begin{array}{c}\text { AAA } \\
\text { Lys }\end{array}$ & $\begin{array}{l}\text { ATT } \\
\text { Ile }\end{array}$ & $\begin{array}{c}\text { GAG } \\
\text { Glu }\end{array}$ & $\begin{array}{c}\text { GCG } \\
\text { Ala }\end{array}$ & $\begin{array}{l}\text { CTT } \\
\text { Leu }\end{array}$ & $\begin{array}{l}\text { TCT } \\
\text { Ser }\end{array}$ & $\begin{array}{l}\text { CAA } \\
\text { Gln }\end{array}$ & $\begin{array}{l}\text { ATG } \\
\text { Met }\end{array}$ & $\begin{array}{c}\text { GAT } \\
\text { Asp }\end{array}$ & $\begin{array}{l}675 \\
225 \\
\end{array}$ \\
\hline $\begin{array}{l}676 \\
226\end{array}$ & $\begin{array}{l}\text { ATT } \\
\text { Ile }\end{array}$ & $\begin{array}{l}\text { CTT } \\
\text { Leu }\end{array}$ & $\begin{array}{l}\text { TTT } \\
\text { Phe }\end{array}$ & $\begin{array}{c}\text { CAT } \\
\text { His }\end{array}$ & $\begin{array}{l}\text { AGT } \\
\text { Ser }\end{array}$ & $\begin{array}{c}\text { AGT } \\
\text { Ser }\end{array}$ & $\begin{array}{c}\text { GAT } \\
\text { Asp }\end{array}$ & $\begin{array}{c}\text { GGA } \\
\text { Gly }\end{array}$ & $\begin{array}{l}\text { ATG } \\
\text { Met }\end{array}$ & $\frac{\text { CTT }}{\text { Leu }}$ & $\begin{array}{c}\text { GTG } \\
\text { Val }\end{array}$ & $\begin{array}{l}\text { CTT } \\
\text { Leu }\end{array}$ & $\begin{array}{l}\text { GAT } \\
\text { Asp }\end{array}$ & $\begin{array}{c}\text { GCT } \\
\text { Ala }\end{array}$ & $\begin{array}{c}\text { GAA } \\
\text { Glu }\end{array}$ & $\begin{array}{l}720 \\
240 \\
\end{array}$ \\
\hline $\begin{array}{l}721 \\
241\end{array}$ & $\begin{array}{l}\text { ACT } \\
\text { Thr }\end{array}$ & $\begin{array}{c}\text { GTG } \\
\text { Val }\end{array}$ & $\begin{array}{l}\text { TGC } \\
\text { Cys }\end{array}$ & $\begin{array}{l}\text { TTA } \\
\text { Leu }\end{array}$ & $\begin{array}{c}\text { CCC } \\
\text { Pro }\end{array}$ & $\begin{array}{c}\text { AAG } \\
\text { Lys }\end{array}$ & $\begin{array}{l}\text { CTG } \\
\text { Leu }\end{array}$ & $\begin{array}{c}\text { GTG } \\
\text { Val }\end{array}$ & $\begin{array}{c}\text { CAG } \\
\text { Gln }\end{array}$ & $\begin{array}{c}\text { GGG } \\
\text { Gly }\end{array}$ & $\begin{array}{l}\text { ACG } \\
\text { Thr }\end{array}$ & $\begin{array}{c}\text { TGG } \\
\text { Trp }\end{array}$ & $\begin{array}{l}\text { GGT } \\
\text { Gly }\end{array}$ & $\begin{array}{c}\text { CCC } \\
\text { Pro }\end{array}$ & $\begin{array}{l}\text { TCT } \\
\text { Ser }\end{array}$ & $\begin{array}{l}765 \\
255\end{array}$ \\
\hline $\begin{array}{l}766 \\
256 \\
\end{array}$ & $\begin{array}{c}\text { GGC } \\
\text { Gly }\end{array}$ & $\begin{array}{c}\text { AGC } \\
\text { Ser }\end{array}$ & $\begin{array}{c}\text { TCA } \\
\text { Ser }\end{array}$ & $\begin{array}{c}\text { CAG } \\
\text { Gln }\end{array}$ & $\begin{array}{c}\text { AGC } \\
\text { Ser }\end{array}$ & $\begin{array}{c}\text { CTC } \\
\text { Leu }\end{array}$ & $\begin{array}{c}\text { TAC } \\
\text { Tyr }\end{array}$ & $\begin{array}{c}\text { GAA } \\
\text { Glu }\end{array}$ & $\begin{array}{c}\text { ATC } \\
\text { Ile }\end{array}$ & $\begin{array}{l}\text { TGT } \\
\text { Cys }\end{array}$ & $\begin{array}{c}\text { GTG } \\
\text { Val } \\
\end{array}$ & $\frac{\text { TGT }}{\text { Cys }}$ & $\begin{array}{c}\text { CCA } \\
\text { Pro } \\
\end{array}$ & $\begin{array}{c}\text { GAT } \\
\text { Asp }\end{array}$ & $\frac{\text { GGG }}{\text { Gly }}$ & $\begin{array}{l}810 \\
270 \\
\end{array}$ \\
\hline $\begin{array}{l}811 \\
271 \\
\end{array}$ & $\begin{array}{l}\text { AAG } \\
\text { Lys }\end{array}$ & $\begin{array}{l}\text { CTG } \\
\text { Leu }\end{array}$ & $\begin{array}{c}\text { TAC } \\
\text { Tyr }\end{array}$ & $\begin{array}{c}\text { CTG } \\
\text { Leu }\end{array}$ & $\begin{array}{l}\text { TCT } \\
\text { Ser }\end{array}$ & $\begin{array}{c}\text { GTG } \\
\text { Val }\end{array}$ & $\begin{array}{c}\text { GCC } \\
\text { Ala } \\
\end{array}$ & $\begin{array}{c}\text { GGT } \\
\text { Gly }\end{array}$ & $\begin{array}{c}\text { GTG } \\
\text { Val }\end{array}$ & $\begin{array}{c}\text { AGC } \\
\text { Ser }\end{array}$ & $\begin{array}{c}\text { ACC } \\
\text { Thr }\end{array}$ & $\begin{array}{c}\text { ACG } \\
\text { Thr }\end{array}$ & $\begin{array}{l}\text { TGC } \\
\text { Cys }\end{array}$ & $\begin{array}{c}\text { CAG } \\
\text { Gln }\end{array}$ & $\begin{array}{c}\text { GAG } \\
\text { Glu }\end{array}$ & $\begin{array}{l}855 \\
285 \\
\end{array}$ \\
\hline $\begin{array}{l}856 \\
286\end{array}$ & $\begin{array}{c}\text { CAC } \\
\text { His }\end{array}$ & $\begin{array}{l}\text { AGC } \\
\text { Ser }\end{array}$ & $\begin{array}{c}\text { CAC } \\
\text { His }\end{array}$ & $\begin{array}{c}\text { ATC } \\
\text { Ile }\end{array}$ & $\begin{array}{l}\text { TGC } \\
\text { Cys }\end{array}$ & $\begin{array}{c}\text { CTC } \\
\text { Leu }\end{array}$ & $\begin{array}{c}\text { TGA } \\
\text { Ter }\end{array}$ & & & & & & & & & \\
\hline
\end{tabular}

${ }^{*}$ ) Note the previously determined missense mutations listed in bold in the gray cells.

The homozygous mutations in these patients were highlighted with dark cells and white letters.

The silent mutations was underlined. 
Immunohistochemical analysis of the sarcolemmal proteins such as dystrophin, SGCs, merosin, and dysferlin is an important part of the diagnostic evaluation of muscle biopsies in patients with muscular dystrophy. Reduced or absent sarcolemmal expression of one of the 4 SGCs can be found in patients with any LGMDs and also in patients with dystrophinopathies. It has been previously suggested that different patterns of SGC expression could predict the primary genetic defect, and that genetic analysis could be directed by these patterns $(1,12)$. However Klinge et al. (10) reported that residual SGC expression could be highly variable and an accurate prediction of the genotype could not be achieved. Therefore they recommended using antibodies against all four SGCs for immunoanalysis of skeletal muscle sections. Similarly, a concomitant reduction of dystrophin and any one of SGCs may have a crucial importance in the differential diagnosis of dystrophinopathies for sarcoglycan deficient LGMD (1-5). For this reason, it is not easy to decide whether the disease is a dystrophinopathy with defective expressions of SGCs or a LGMD with defective expression of dystrophin. Since in the cases of this series, the sarcolemmal dystrophin staining and dystrophin gene were not abnormal, Duchenne Muscular Dystrophy (DMD) or Becker Muscular Dystrophy (BMD) were not considered for differential diagnosis.

Patients with any LGMD may be clinically indistinguishable from those with primary dystrophinopathies. Probably, the diagnosis of LGMD has been underestimated and a number of male patients were diagnosed as DMD or $\operatorname{BMD}(1,3,7)$. If a definitive diagnosis can be made based on appropriate immunohistochemical examinations and molecular analysis performed in those patients, a normal staining pattern of dystrophin and an autosomal recessive mode of inheritance can be determined. On the contrary, patients with dystrophinopathy may show variable findings from normal to regional absence or mosaic pattern of sarcolemmal staining with anti-SGCs antibodies which signify different presentation of abnormal organization of the cellmembrane associated dystrophin glycoprotein complex. Therefore careful examination of immunohistochemical staining with genetic study is necessary to make an accurate diagnosis $(1,2)$.

In summary, this study adds different mutations to the growing list of defects that can be associated with LGMD2C and further emphasizes the importance of systematic analysis of all related genes, instead of analyzing only the primarily deficient SGCs gene. In this study, we have also highlighted the patterns of genetic complexity associated with LGMD-2C encountered during the process of differential diagnosis of muscular dystrophies (18).

\section{CONFLICT of INTEREST}

The authors had received financial support from Izmir Katip Çelebi University.

\section{REFERENCES}

1. Dubowitz V, Sewry CA, Oldfors A. Muscle Biopsy: A practical approach. Philadelphia: Saunders; 2013.276-302.

2. Sandonà D, Betto R. Sarcoglycanopathies: Molecular pathogenesis and therapeutic prospects. Expert Rev Mol Med. 2009;11:e28.

3. Ben Jelloun-Dellagi S, Chaffey P, Hentati F, Ben Hamida C, Tome F, Colin H, Dellagi K, Kaplan JC, Fardeau M, Ben Hamida M. Presence of normal dystrophin in Tunisian severe childhood autosomal recessive muscular dystrophy. Neurology. 1990;40:1903.

4. Pogue R, Anderson LV, Pyle A, Sewry C, Pollitt C, Johnson MA, Davison K, Moss JA, Mercuri E, Muntoni F, Bushby KM. Strategy for mutation analysis in the autosomal recessive limb-girdle muscular dystrophies. Neuromuscul Disord. 2001;11:80-7.

5. Moreira ES, Vainzof M, Suzuki OT, Pavanello RC, Zatz M, PassosBueno MR. Genotype-phenotype correlations in 35 Brazilian families with sarcoglycanopathies including the description of three novel mutations. J Med Genet. 2003;40:E12.

6. Gulati S, Leekha S, Sharma MC, Kalra V. Gamma-sarcoglycanopathy. Indian Pediatr. 2003;40:1077-81.

7. Azibi K, Bachner L, Beckmann J S, Matsumura K, Hamouda E, Chaouch M, Chaouch A, Ait-Ouarab R, Vignal A, Weissenbach J. Severe childhood autosomal recessive muscular dystrophy with the deficiency of the $50 \mathrm{kDa}$ dystrophin-associated glycoprotein maps to chromosome 13q12. Hum Molec Genet. 1993;2:1423-28.

8. El Kerch F, Sefiani A, Azibi K, Boutaleb N, Yahyaoui M, Bentahila A, Vinet MC, Leturcq F, Bachner L, Beckmann J, et al. Linkage analysis of families with severe childhood autosomal recessive muscular dystrophy in Morocco indicates genetic homogeneity of the disease in North Africa. J Med Genet. 1994;31: 342-3.

9. Lasa A, Piccolo F, de Diego C, Jeanpierre M, Colomer J, Rodríguez MJ, Urtizberea JA, Baiget M, Kaplan J, Gallano P. Severe limb girdle muscular dystrophy in Spanish gypsies: Further evidence for a founder mutation in the gamma-sarcoglycan gene. Eur J Hum Genet. 1998;6:396-9.

10. Klinge L, Dekomien G, Aboumousa A, Charlton R, Epplen JT, Barresi R, Bushby K, Straub V. Sarcoglycanopathies: Can muscle immunoanalysis predict the genotype? Neuromuscul Disord. 2008;18:934-41.

11. Trabelsi M, Kavian N, Daoud F, Commere V, Deburgrave N, Beugnet C, Llense S, Barbot JC, Vasson A, Kaplan JC, Leturcq F, Chelly J. Revised spectrum of mutations in sarcoglycanopathies. Eur J Hum Genet. 2008;16:793-803.

12. Ferreira AF, Carvalho MS, Resende MB, Wakamatsu A, Reed UC, Marie SK. Phenotypic and immunohistochemical characterization of sarcoglycanopathies. Clinics (Sao Paulo). 2011;66:1713-19. 
13. Jung D, Leturcq F, Sunada Y, Duclos F, Tome FMS, Moomaw C, Merlini L, Azibi K, Chaouch M, Slaughter C, Fardeau M, Kaplan JC, Campbell KP. Absence of gamma-sarcoglycan (35 DAG) in autosomal recessive muscular dystrophy linked to chromosome 13q12. FEBS Lett. 1996:381: 15-20.

14. Kefi M, Amouri R, Driss A, Ben Hamida C, Ben Hamida M, Kunkel LM, Hentati F. Phenotype and sarcoglycan expression in Tunisian LGMD 2C patients sharing the same del521-T mutation. Neuromuscul Disord. 2003;13:779-87.

15. Navarro C, Teijeira S. Neuromuscular disorders in the Gypsy ethnic group: A short review. Acta Myol. 2003; 22: 11-14.

16. McNally EM, Duggan D, Gorospe J R, Bonnemann C G, Fanin M, Pegoraro E, Lidov HG, Noguchi S, Ozawa E, Finkel RS, Cruse RP, Angelini C, Kunkel LM, Hoffman EP. Mutations that disrupt the carboxyl-terminus of gamma-sarcoglycan cause muscular dystrophy. Hum Molec Genet. 1996;5:1841-7.
17. Merlini L, Kaplan JC, Navarro C, Barois A, Bonneau D, Brasa J, Echenne B, Gallano P, Jarre L, Jeanpierre M, Kalaydjieva L, Leturcq F, Levi-Gomes A, Toutain A, Tournev I, Urtizberea A, Vallat JM, Voit T, Warter JM. Homogeneous phenotype of the gypsy limb-girdle MD with the gamma-sarcoglycan C283Y mutation. Neurology. 2000;54:1075-9.

18. Dinçer P, Akçören Z, Demir E, Richard I, Sancak O, Kale G, Ozme S, Karaduman A, Tan E, Urtizberea JA, Beckmann JS, Topaloğlu $\mathrm{H}$. A cross section of autosomal recessive limb-girdle muscular dystrophies in 38 families. J Med Genet. 2000;37:361-7. 